\title{
Diagnostic Yield of Echocardiography in Syncope Patients with Normal ECG
}

\author{
Nai-Lun Chang, ${ }^{1}$ Priyank Shah, ${ }^{2}$ Sharad Bajaj, ${ }^{2}$ Hartaj Virk, \\ Mahesh Bikkina, ${ }^{2}$ and Fayez Shamoon ${ }^{2}$ \\ ${ }^{1}$ Department of Internal Medicine, St. Joseph's Regional Medical Center, Paterson, NJ 07503, USA \\ ${ }^{2}$ Department of Cardiology, St. Joseph's Regional Medical Center, Paterson, NJ 07503, USA
}

Correspondence should be addressed to Nai-Lun Chang; nailun.chang@gmail.com

Received 23 October 2015; Revised 15 December 2015; Accepted 17 December 2015

Academic Editor: Firat Duru

Copyright (C) 2016 Nai-Lun Chang et al. This is an open access article distributed under the Creative Commons Attribution License, which permits unrestricted use, distribution, and reproduction in any medium, provided the original work is properly cited.

\begin{abstract}
Aim. This study aimed to assess the role of echocardiography as a diagnostic tool in evaluating syncope patients with normal versus abnormal electrocardiogram. Methods. We conducted a retrospective study of 468 patients who were admitted with syncope in 2011 at St. Joseph's Regional Medical Center, Paterson, NJ. Hospital records and patient charts, including initial emergency room history and physical, were carefully reviewed. Patients were separated into normal versus abnormal electrocardiogram groups and then further divided as normal versus abnormal echocardiogram groups. Causes of syncope were extrapolated after reviewing all test results and records of consultations. Results. Three hundred twelve of the total patients (68.6\%) had normal ECG. Twothirds of those patients had echocardiograms; 11 patients (5.7\%) had abnormal echo results. Of the aforementioned patients, three patients had previous documented history of severe aortic stenosis on prior echocardiograms. The remaining eight had abnormal but nondiagnostic echocardiographic findings. Echocardiography was done in 93 of 147 patients with abnormal ECG (63.2\%). Echo was abnormal in 27 patients (29\%), and the findings were diagnostic in 6.5\% patients. Conclusions. This study demonstrates that echocardiogram was not helpful in establishing a diagnosis of syncope in patients with normal ECG and normal physical examination.
\end{abstract}

\section{Introduction}

Syncope, a common clinical syndrome, is characterized by transient loss of consciousness and postural tone caused by brief global cerebral hypoperfusion from decreased cardiac output and/or peripheral resistance. It is of rapid onset, brief duration, and spontaneous and complete recovery. Syncope accounts for about $1 \%$ of all hospital admissions and 3\% emergency department visits and annually costs approximately $\$ 2$ billion in the United States [1-4].

Causes of syncope are vast and often present as a diagnostic challenge for physicians, resulting in extensive workup. The causes could be vascular, cardiac, neurologic, metabolic, psychologic, and finally of unknown origin. As per Linzer et al., the most common causes for syncope are unknown origin (34\%), vasovagal and orthostatic hypotension $(26 \%)$, and arrhythmias (14\%) [5]. Several guidelines exist to assist physicians approach the evaluation of syncope in an algorithmic way [6-8]. Echocardiography (Echo) is a common imaging modality used in the workup of patients presenting with syncope. According to American College of Cardiology 2011 Appropriate Use Criteria for Echocardiography, echo is not necessary in patients with lightheadedness and/or presyncope who do not have history of cardiac disease. However, in syncope patients who have no previous heart disease, the use of echocardiography as one of the diagnostic tools is still appropriate [9]. In 1995, Recchia and Barzilai conducted a retrospective review at an urban university hospital. They considered the following ECG changes as significant: Q waves suggesting prior myocardial infarcts, bundle branch block, ventricular ectopy or arrhythmia, and Mobitz II or higher degrees of atrioventricular nodal block. From chart review of 128 syncope patients, more than $60 \%$ of the patients underwent echocardiography and more than half 
TABLE 1: Definitions of abnormal ECG and echo.

\begin{tabular}{ll}
\hline Abnormal ECG & Abnormal echo \\
\hline (i) Arrhythmias & \\
(ii) Q waves & (i) LVEF < 45\% \\
(iii) Ischemic changes & (ii) Moderate-severe valvular abnormalities (stenosis or regurgitation) \\
(iv) 2nd- and 3rd-degree AV block & (iii) Severe ventricular wall hypertrophy \\
(v) Paced rhythm & (iv) Any septal wall motion abnormality \\
(vi) QTc $>500 \mathrm{~ms}$ & (v) Hypertrophic cardiomyopathy with outflow tract obstruction \\
(vii) Left bundle branch block & (vi) Severe pulmonary hypertension \\
(viii) Bifascicular block & (vii) Hemodynamically significant pericardial effusions \\
(ix) Brugada pattern & \\
(x) Abnormal axis &
\end{tabular}

$\mathrm{AV}$, atrioventricular. LVEF, left ventricular ejection fraction.

the results were normal. No echo reports showed unsuspected causes of syncope. They concluded that echocardiogram did not add to any additional diagnostic value for patients without suspected cardiac disease [10]. Another retrospective study of 323 patients by Anderson et al. in 2012 suggested that echo may be a poor utilization of resources for patients with normal ECG due to very low yield of structural heart disease in such patients. Eighty-eight percent of the 267 patients with normal ECG had echocardiogram done to evaluate cardiac structure. However, none showed structural abnormality [11].

Echocardiography seems to have over time become a routine test ordered to assess a wide array of cardiac diseases. Several studies have shown that echocardiogram has been performed in more than half of the patients with complaints of syncope $[10,12]$. We suggest that the most important diagnostic tool in evaluation of syncope is a detailed history and physical exam. The primary objective of our study was to determine the diagnostic yield of echo in patients presenting with syncope, particularly in those with normal electrocardiogram and physical exam not suggestive of cardiac disease.

\section{Methods}

This is a retrospective, observational study at St. Joseph's Regional Medical Center, a quaternary academic care and state-designated trauma center in Paterson, New Jersey. Data were collected through a retrospective chart review of all adult patients admitted for syncope during a 12-month period from January 2011 to December 2011. Cases were identified based on the initial diagnosis made by physician on admission. We collected and reviewed the following data from medical charts and electronic medical records: history of presenting illness and associated symptoms, demographic data, medical history, and results of ECG and echocardiogram.

The initial ECG was performed on admission. We defined abnormal ECG and echo as the following (Table 1). ECGs showing arrhythmias, Q waves, ischemic changes, 2nd- and 3rd-degree AV block, paced rhythm, QTc > $500 \mathrm{~ms}$, left bundle branch block, bifascicular block, Brugada pattern, and abnormal axis were considered abnormal. All echocardiograms were performed by registered diagnostic sonographers. Echocardiograms demonstrating ejection fraction less than $45 \%$, any severe valvular abnormalities, severe ventricular wall hypertrophy, septal wall motion abnormality, hypertrophic cardiomyopathy with outflow tract obstruction, severe pulmonary hypertension, and hemodynamically significant pericardial effusions were considered abnormal.

Both ECG and echo reports were read and approved by an attending cardiologist. The reports were then reviewed by investigators.

Initial history and physical exam in the emergency room and the results of all diagnostic tests and records of all consultations were reviewed. The final cause of syncope was assigned after careful review of all physicians' notes and test results. We looked to see the number of patients admitted with syncope who had ECG and echocardiogram and the number of patients who had abnormal ECG and/or abnormal echo.

\section{Results}

Out of the 468 patients, we had 321 patients (68.6\%) with normal ECG. One hundred ninety-two echocardiograms (59.8\%) were done in patients with normal ECG reading (Figure 1). Of those, 11 patients (5.7\%) had abnormal TTE. However, 3 patients had prior documented echo results and history and physical findings of severe aortic stenosis. Discharge diagnoses were orthostatic hypotension and unknown causes. In comparison, in the group of 147 patients $(31.4 \%)$ with abnormal ECG, 93 echocardiograms (63.2\%) were done, and 27 of them (29\%) were abnormal. However, the finding of structural abnormalities on echo does not establish the cause for the patient's syncopal episode.

Echocardiogram by itself did not help in determining the cause of syncope in any patient in normal ECG group. Three of the eleven patients with abnormal echocardiograms had prior documentation of severe aortic stenosis. The other eight patients, which are $4 \%$ of the patients with normal ECG with abnormal TTE, had abnormal echo findings showing reduced LVEF less than $45 \%$ or other nonaortic valvular pathology. Two patients had vasovagal response; one patient had orthostatic hypotension. Five patients had unknown cause upon chart review (Table 2). However, echo did not help in establishing diagnosis in any of those patients. 


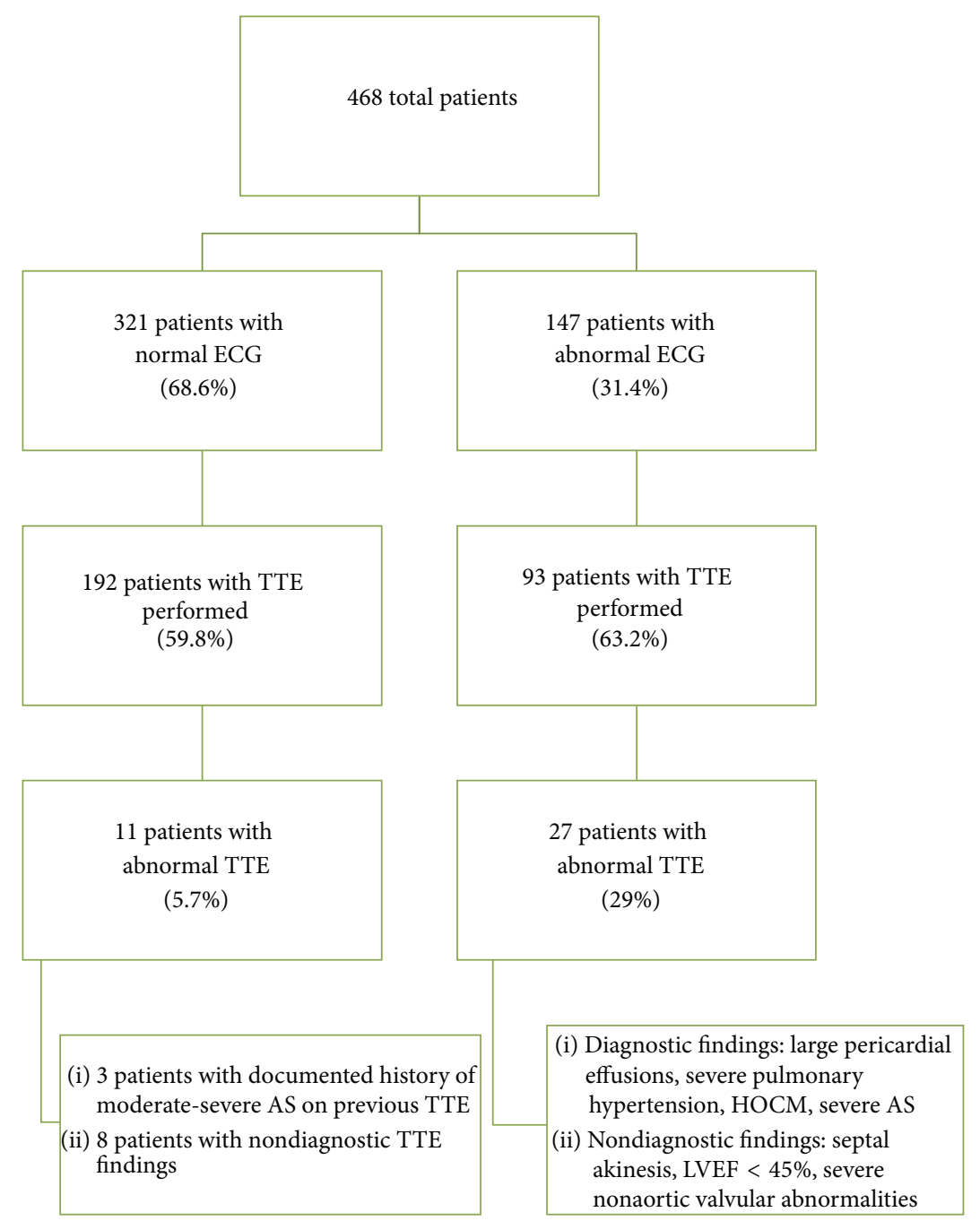

FIGURE 1: Patient segregation flowchart. ECG, electrocardiogram. TTE, transthoracic echocardiogram. HOCM, hypertrophic cardiomyopathy. AS, aortic stenosis.

Of the abnormal echocardiograms in the abnormal ECG group, besides the nondiagnostic findings, there were one patient with severe pulmonary hypertension, two patients with significant pericardial effusions, one patient with severe aortic stenosis, one patient with significant septal motion abnormality, and one with hypertrophic cardiomyopathy. Those are all diagnostic causes of syncope (Table 2). Hence, in the abnormal ECG group, echo aided in the diagnosis in $6.5 \%$ patients (6 out of 93 ).

In patients with TTE performed, nonspecific causes, vasovagal response, and orthostatic hypotension all together comprise $91.6 \%$ and $68.8 \%$ of the discharge diagnosis in normal ECG and abnormal ECG groups, respectively (Table 3).

In the normal ECG group, the six patients found to have arrhythmia (i.e., paroxysmal atrial fibrillation) or conduction disease (i.e., sinus node dysfunction and severe His-Purkinje conduction disease) as possible causes of their syncopal episode, echocardiogram did not show significant cardiac structural abnormalities.

\section{Discussion}

In clinical practice, echocardiography has become one of the most routinely ordered modalities for assessing the cause of presyncope and syncope. Recchia and Barzilai [10] found that echocardiography was performed in more than half of the patients with complaints of syncope but no finding had any diagnostic value. Several studies have shown that younger patients, patients with no significant cardiac history, have low echocardiographic yield. Panther et al. [13] demonstrated in a 7-year retrospective study of 439 patients, who were referred for echocardiographic studies in evaluation of syncope, patients younger than 40 years of age with syncope will most likely have normal echocardiography. In age groups $40-59$ and $60+, 58.2 \%$ and $29.6 \%$ had normal echocardiographic findings. In a prospective, observational study done by Sarasin et al. [14] in 2002, 155 of 650 patients (23\%) presented with syncope without clear cause. Routine echocardiography showed no diagnostic cause to explain 


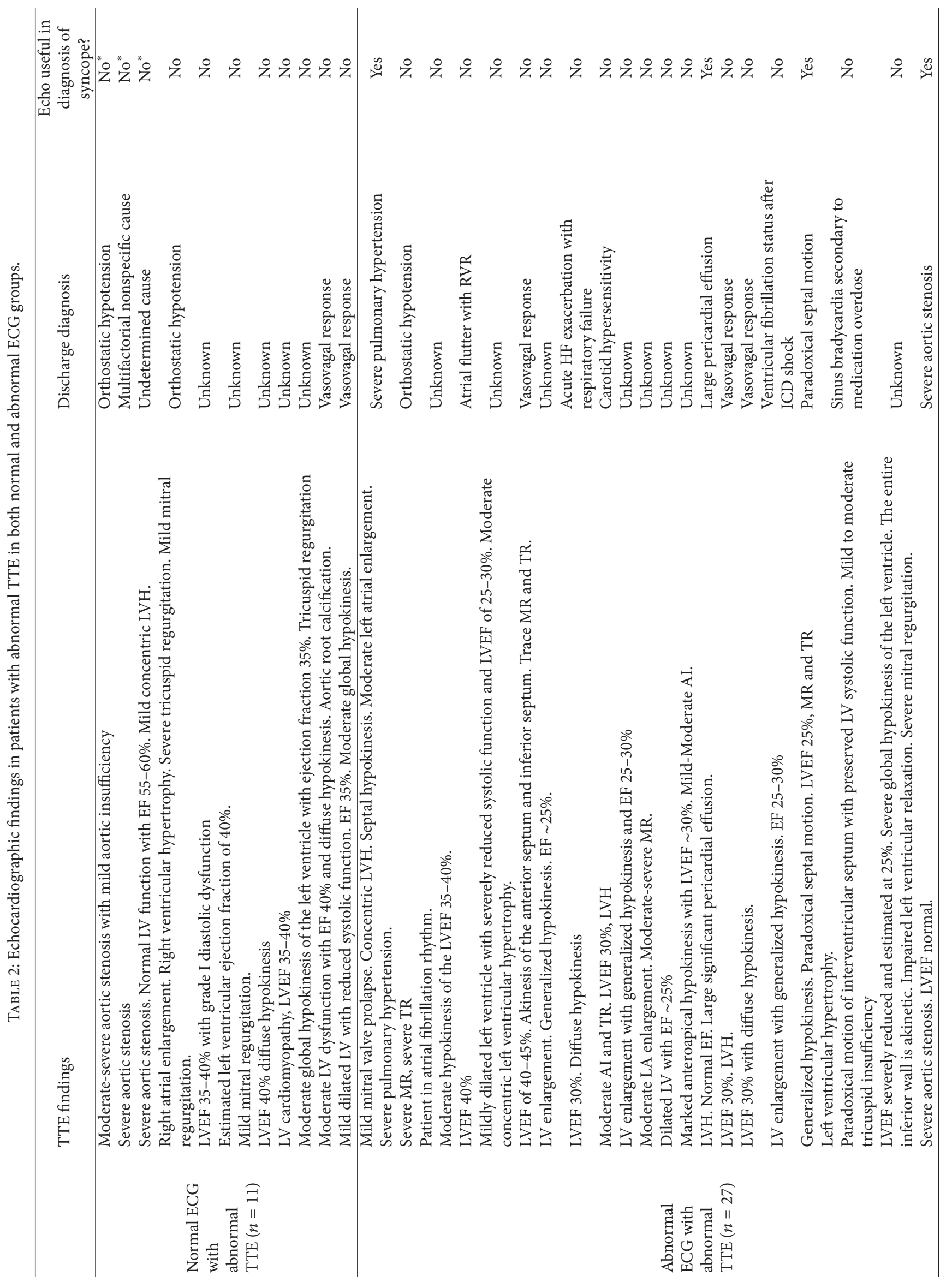




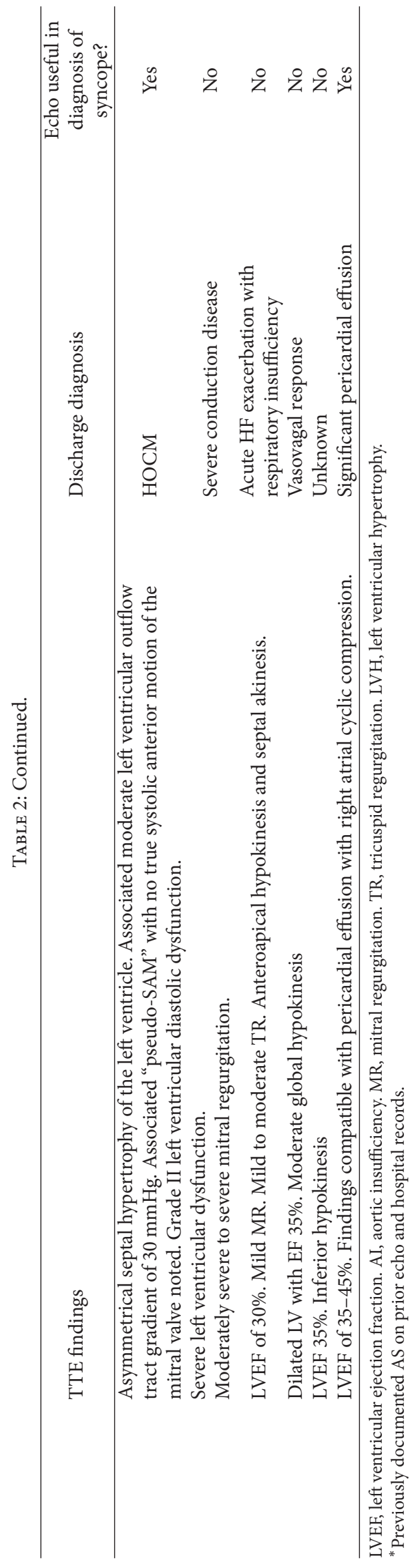


TABLE 3: Causes of syncope in normal and abnormal ECG groups with TTE performed.

\begin{tabular}{lcc}
\hline Cause of syncope & Normal ECG with TTE performed (\%) & Abnormal ECG with TTE performed (\%) \\
\hline Vasovagal/orthostatic & $58(30.2)$ & $32(34.4)$ \\
Arrhythmia/conduction diseases & $6(3.13)$ & $16(17.2)$ \\
Symptomatic anemia & $1(0.5)$ & 0 \\
TIA/CVA & $3(1.5)$ & 0 \\
Any severe valvular diseases & $3(1.5)$ & $1(1.08)$ \\
Ischemic cardiac events/acute HF exacerbation & $3(1.5)$ & $10(10.8)$ \\
Device malfunction & 0 & $1(1.08)$ \\
Nonspecific & $118(61.5)$ & $32(34.4)$ \\
Carotid hypersensitivity & 0 & $1(1.08)$ \\
Total & 192 & 93 \\
\hline
\end{tabular}

TIA, transient ischemia attack. CVA, cerebrovascular accidents. HF, heart failure.

the event. In patients without significant cardiac history or abnormal ECG, echocardiography was nondiagnostic or normal. Thirteen percent of the patients had positive cardiac history or abnormal ECG but echocardiogram only showed left ventricular systolic dysfunction or minor nonrelevant findings. They concluded that echocardiography was most useful when its use was restricted to high-risk patients with significant history of cardiac disease and ECG abnormalities.

Results of our study prove that echocardiogram has a low overall diagnostic yield in syncope patients without a history of cardiac structural disease. Eleven patients with normal ECG (5.7\%) had abnormal TTE. Three of those already had previous documentation of severe aortic stenosis on prior history and physical exam and echocardiograms. Remaining eight patients' echocardiogram did not uncover any intracardiac abnormality to have caused the syncopal episode. Hence the diagnostic value of echo in patients with normal ECG and normal physical exam was $0 \%$. For patients with abnormal ECG, echo aided in diagnosis in $6.5 \%$ patients. Majority of the patients from either group had discharge diagnosis as syncope due to nonspecific or undetermined causes.

In agreement with other similar studies, we reason that echocardiography should not be routinely used in patients presenting with syncope particularly in those with normal ECG and physical exam not suggestive of cardiac disease. Echocardiogram on every patient that comes with complaints of syncope is not cost effective.

\section{Limitations}

For one, this is a retrospective study. We do not know the outcomes of the patients after their discharge. Second, 129 of the 321 patients (40\%) from the normal ECG group did not have an echocardiogram done on that admission. If echocardiogram was done, the percentage of abnormal finding may be higher. Third, we depended on emergency room records for the initial history and physical examination. Details could have been missed. Reviewing of the charts further strengthens the call for more detailed history and physical exam upon initial contact of patients.

\section{Conclusions}

Echocardiography should not be a routine diagnostic tool used to elucidate causes of syncope unless causes were unexplained by history and physical exam and patients have significant cardiac history with abnormal ECG.

\section{Conflict of Interests}

The authors declare that there is no conflict of interests regarding the publication of this paper.

\section{References}

[1] H. Calkins and D. P. Zipes, "Hypotension and syncope," in Braunwald's Heart Disease, vol. 1, pp. 885-895, Saunders, 9th edition, 2012.

[2] W. N. Kapoor, "Evaluation and management of the patient with syncope," The Journal of the American Medical Association, vol. 268, no. 18, pp. 2553-2560, 1992.

[3] B. C. Sun, J. A. Emond, and C. A. Camargo Jr., "Direct medical costs of syncope-related hospitalizations in the United States," American Journal of Cardiology, vol. 95, no. 5, pp. 668-671, 2005.

[4] H. Calkins, M. Byrne, R. El-Atassi, S. Kalbfleisch, J. J. Langberg, and F. Morady, "The economic burden of unrecognized vasodepressor syncope," The American Journal of Medicine, vol. 95, no. 5, pp. 473-479, 1993.

[5] M. Linzer, E. H. Yang, N. A. M. Estes III, P. Wang, V. R. Vorperian, and W. N. Kapoor, "Diagnosing syncope: part 1: value of history, physical examination, and electrocardiography," Annals of Internal Medicine, vol. 126, no. 12, pp. 989-996, 1997.

[6] M. Brignole, P. Alboni, D. G. Benditt et al., "Guidelines on management (diagnosis and treatment) of syncope-update 2004. Executive summary," European Heart Journal, vol. 2, pp. 2054-2072, 2004.

[7] A. Moya, R. Sutton, F. Ammirati et al., "Guidelines for the diagnosis and management of syncope (version 2009): Task Force for the Diagnosis and Management of Syncope, European Society of Cardiology (ESC), European Heart Rhythm Association (EHRA)," European Heart Journal, vol. 30, no. 21, pp. 26312671, 2009.

[8] S. A. Strickberger, D. W. Benson, I. Biaggioni et al., "AHA/ACCF scientific statement on the evaluation of syncope: from the 
American Heart Association Councils on Clinical Cardiology, Cardiovascular Nursing, Cardiovascular Disease in the Young, and Stroke, and the Quality of Care and Outcomes Research Interdisciplinary Working Group; and the American College of Cardiology Foundation: in collaboration with the Heart Rhythm Society: endorsed by the American Autonomic Society," Circulation, vol. 113, pp. 316-327, 2006.

[9] P. S. Douglas, M. J. Garcia, D. E. Haines et al., "ACCF/ASE/AHA/ ASNC/HFSA/HRS/SCAI/SCCM/SCCT/ SCMR 2011 appropriate use criteria for echocardiography: a report of the American College of Cardiology Foundation Appropriate use Criteria Task Force, American Society of Echocardiography, American Heart Association, American Society of Nuclear Cardiology, Heart Failure Society of America, Heart Rhythm Society, Society for Cardiovascular Angiography and Interventions, Society of Critical Care Medicine, Society of Cardiovascular Computed Tomography, and Society for Cardiovascular Magnetic Resonance," Journal of the American College of Cardiology, vol. 57, pp. 1126-1166, 2011.

[10] D. Recchia and B. Barzilai, "Echocardiography in the evaluation of patients with syncope," Journal of General Internal Medicine, vol. 10, no. 12, pp. 649-655, 1995.

[11] K. L. Anderson, A. Limkakeng, E. Damuth, and A. Chandra, "Cardiac evaluation for structural abnormalities may not be required in patients presenting with syncope and a normal ECG result in an observation unit setting," Annals of Emergency Medicine, vol. 60, no. 4, pp. 478-484.el, 2012.

[12] H. Calkins, M. Byrne, R. El-Atassi, S. Kalbfleisch, J. J. Langberg, and F. Morady, "The economic burden of unrecognized vasodepressor syncope," The American Journal of Medicine, vol. 95, no. 5, pp. 473-479, 1993.

[13] R. Panther, S. Mahmood, and R. Gal, "Echocardiography in the diagnostic evaluation of syncope," Journal of the American Society of Echocardiography, vol. 11, no. 3, pp. 294-298, 1998.

[14] F. P. Sarasin, A.-F. Junod, D. Carballo, S. Slama, P.-F. Unger, and M. Louis-Simonet, "Role of echocardiography in the evaluation of syncope: a prospective study," Heart, vol. 88, no. 4, pp. 363$367,2002$. 


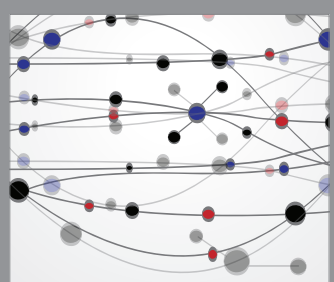

The Scientific World Journal
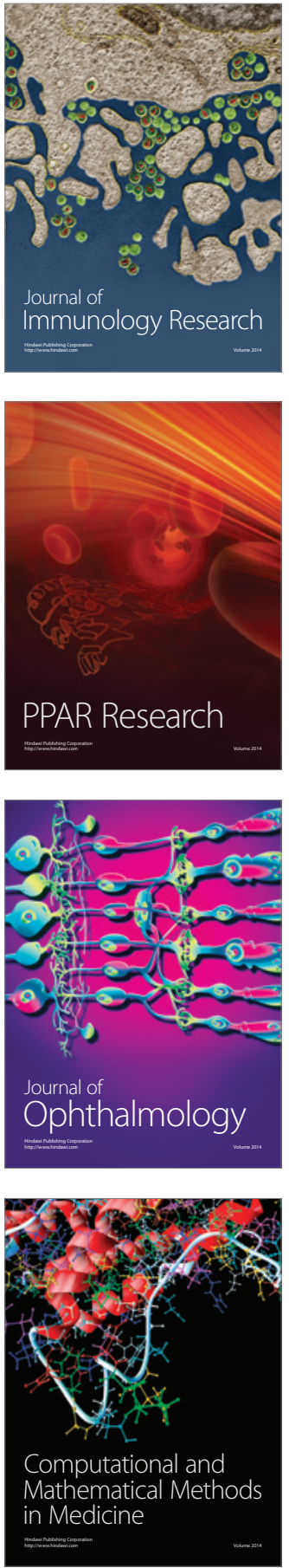

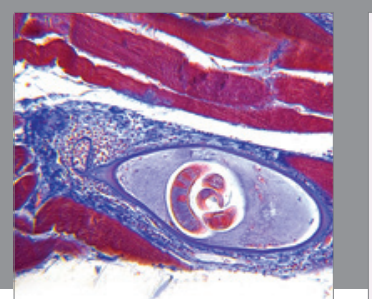

Gastroenterology Research and Practice

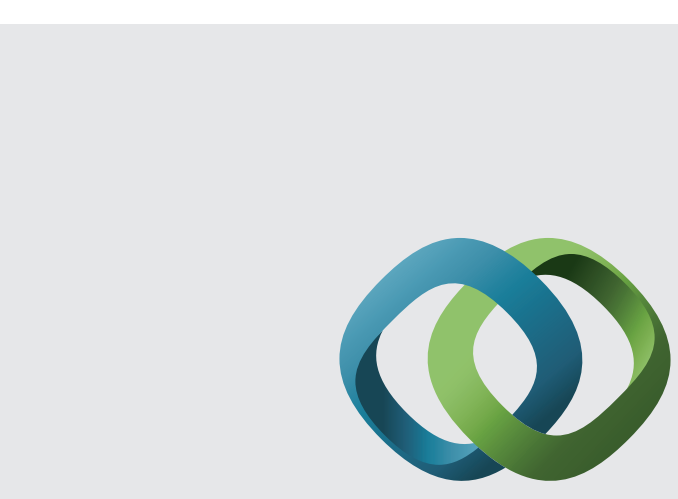

\section{Hindawi}

Submit your manuscripts at

http://www.hindawi.com
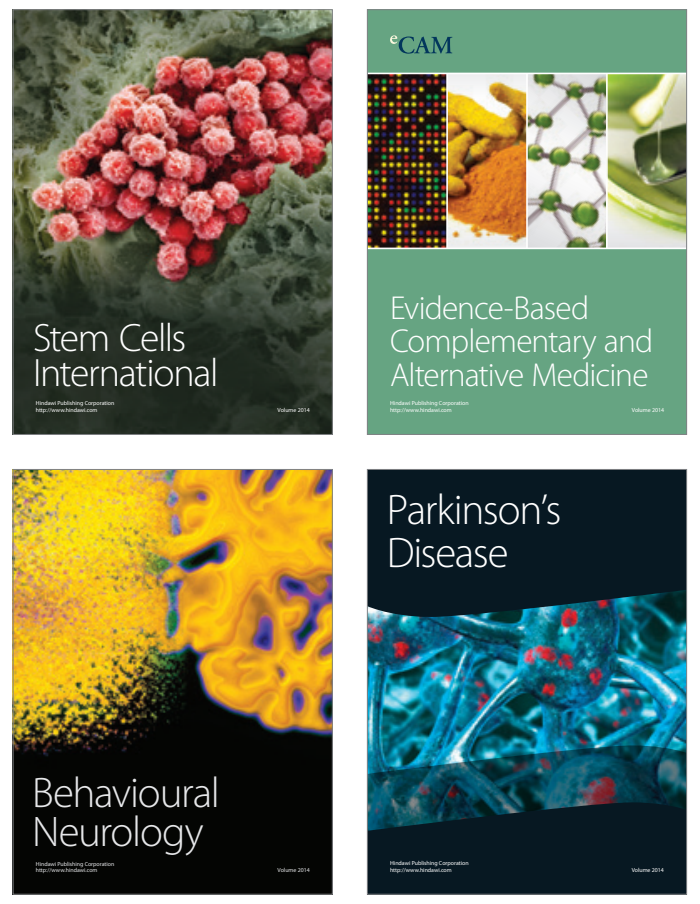
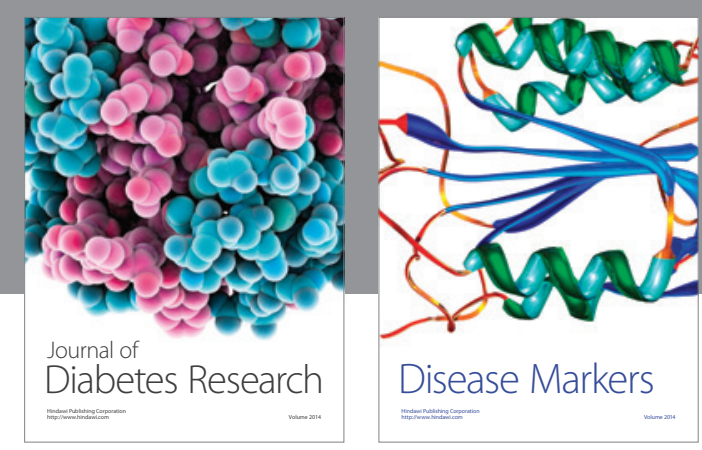

Disease Markers
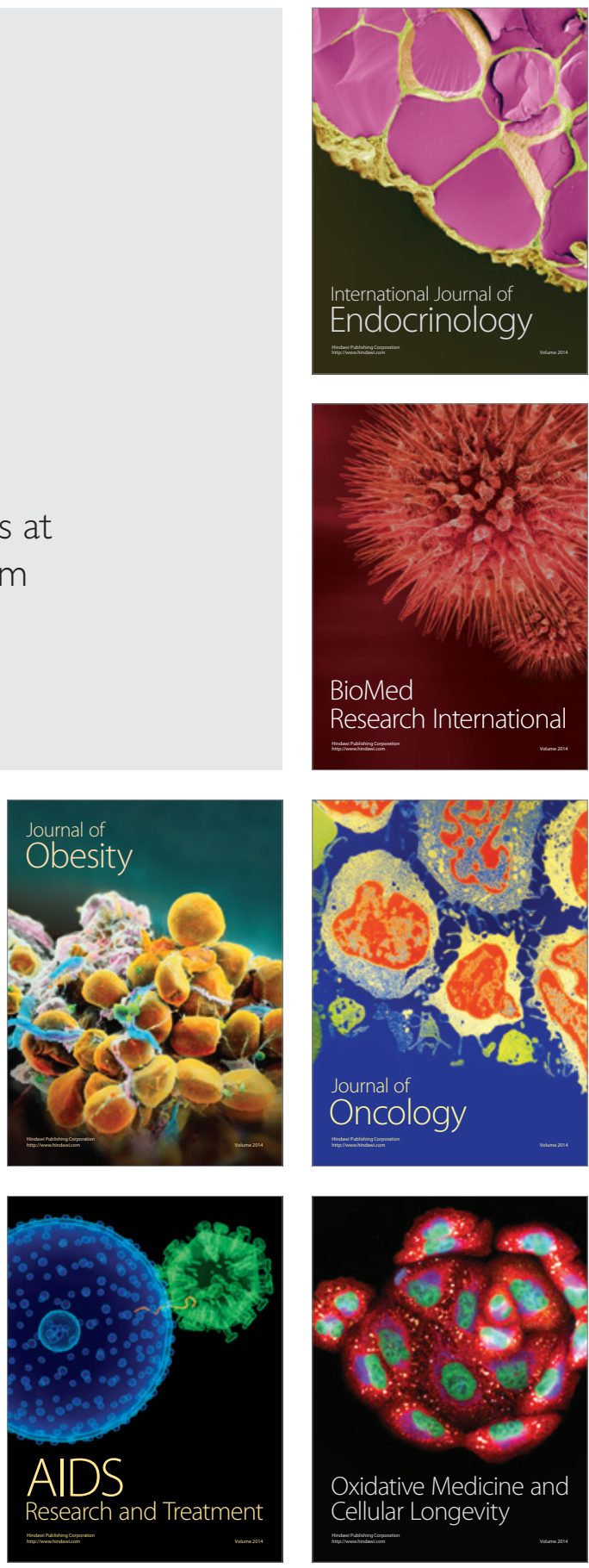